\title{
Approximation in Weighted Orlicz Spaces with a generating Young function that might be non- convex
}

\author{
Ali DOĞU',*, Yunus Emre YILDIRIR ${ }^{2}$ \\ ${ }^{1}$ Ballkesir University, Institute of Science, Cagis Campus, Balikesir \\ ${ }^{2}$ Ballkesir University Necatibey Faculty of Education, Balikesir \\ Gelis Tarihi (Received Date): 27.01.2021 \\ Kabul Tarihi (Accepted Date): 09.06.2021
}

\begin{abstract}
The aim of this paper is to investigate the order of approximation by some linear summation methods of trigonometric Fourier series in weighted Orlicz spaces which have generating Young functions not necessary to be convex. Obtained estimations base on the fractional modulus of smoothness and the best approximation. Furthermore, a convolution type operator is defined and its estimation by the best approximation is obtained.
\end{abstract}

Keywords: Linear summation processes, Fourier series, trigonometric approximation, weighted Orlicz spaces, Muckenhoupt weight.

\section{Konveks olması gerekmeyen genelleştirilmiş Young fonksiyonu ile üretilen Ağırlıklı Orlicz Uzaylarında yaklaşım}

$\ddot{\mathbf{O} z}$

Bu çalışmada, konveks olması gerekmeyen Young fonksiyonları ile üretilen ağırlıklı Orlicz uzaylarında trigonometrik Fourier serilerinin bazı lineer toplam metodları ile yaklaşım problemleri incelenmiştir. Elde edilen sonuçlar kesirli düzgünlük modülüne ve en iyi yaklaşım sayısına dayanmaktadır. Ayrıca, konvolüsyon tipli dönüşüm tanımlayıp, bu dönüşüm ile en iyi yaklaşım sayısı arasındaki ilişki değerlendirilmiştir.

\footnotetext{
* Ali DOĞU, dogualii19831227@ gmail.com, https://orcid.org/0000-0001-7848-4891

Yunus Emre YILDIRIR, yildirir@balikesir.edu.tr, http://orcid.org/0000-0001-5526-4263
} 
Anahtar kelimeler: Lineer toplam metodları, Fourier serileri, trigonometrik yaklaşım, ă̆ırlıklı Orlicz uzayları, Muckenhoupt ă̆ırlık fonksiyonu.

\section{Introduction}

The concept of Orlicz space expands the well-known concept of the space $L_{p}, p \geq 1$. The function $t^{p}$ in the defnition of the space $L_{p}$ is replaced by a more general convex function which is called a Young function. Further information about Orlicz space can be found in [11, 17]. Many applications of Orlicz spaces have emerged [16]. On the other hand, in the paper [3], a diferent approach to the Orlicz space appeared. In this study, Chen generalized the definition of Orlicz space saving almost all known properties of this space. In this generalization, Young function does not have to be convex. Later, in [1] this approach was developed with Muckenhoupt weights and was proved direct and inverse trigonometric approximation theorems in these spaces by Akgun. For the other papers about trigonometric approximation in these spaces, see [2, $12,22,4,23]$. We firstly introduce this space.

We denote by $\Phi$ the class of the strictly increasing functions $\phi:[0, \infty) \rightarrow[0, \infty)$ such that $\phi(\infty)=\infty$. Let $N[p, q]$ be denote the class of even functions $\varphi \in \Phi$ such that $\varphi(x) x^{-p}$ is non-decreasing and $\varphi(x) \cdot x^{-q}$ is non-increasing when $|x|$ is increasing $(0, \infty)$. By $N<p, q>$, we denote the class of function $\varphi$ in $N[p+\varepsilon, q-\delta]$ for some small number $\varepsilon, \delta>0$. Suppose that $\Phi_{\mathrm{p}}$ denotes the class of function $M$ in $N\langle p, q\rangle$ for some $1<p \leq q<\infty$. We say that the function $M$ satifies the condition $\Delta_{2}$ if there exists a constant $c>0$ and $u_{0}>0$ such that $M(2 u) \leq c M(u)$ for $u \geq u_{0}$. In this case, we denote $M \in \Delta_{2}$. The functions in $\Phi_{\mathrm{p}}, \mathrm{p}>1$, are continuous and satisfy the conditions $\mathrm{M}(0)=0$ and $M \in \Delta_{2}$. These functions may not be convex [3, p. 67-68].

Let $T=[-\pi, \pi]$. We say a nonnegative function $\omega$ is a weight function if it is measurable and positive almost everywhere on $T$.

Let $\quad M \in \Phi_{p}, \quad p>1$ and $\omega$ be a weight function on $T$. We define $\varphi_{M}(t):=M(t) / t$. Since $1<p<\infty$, we get $\varphi_{M}(t) \rightarrow \infty$ as $t \rightarrow \infty$. Let $\psi_{M}(t)$ be denote the inverse function of positive non-decreasing continuous function $\varphi_{M}(t)$. We set

$\Phi_{M}(x)=\int_{0}^{x} \varphi_{M}(t) d t$

and

$\Psi_{M}(x)=\int_{0}^{x} \psi_{M}(t) d t$

$\Phi_{M}$ is a convex function and so $\Psi_{M}(x)$ is the complementary function of $\Phi_{M}$, in the sense of Young. We define the weighted Orlicz space as

$L_{M, \omega}(T)=\left\{f: T \rightarrow R: f\right.$ measureable function and $\left.\int_{T} \Phi_{M}(|f(x)|) \omega(x) d x<\infty\right\}$ 
On this space, we define Orlicz norm as

$\|f\|_{M, \omega}:=\sup _{g}\left\{\int_{T}(|f(x) g(x)|) \omega(x) d x: \int_{T} \Psi_{M}(|g(x)|) \omega(x) d x \leq 1\right\}$

and the Luxemburg norm

$\|f\|_{(M), \omega}:=\inf \left\{k>0: \int_{T} \Phi_{M}\left(k^{-1}|f(x)|\right) \omega(x) d x \leq 1\right\}$.

The equivalence

$\|f\|_{(M), \omega} \sim\|f\|_{M, \omega}$

is valid [1].

It is seen that $L_{M, \omega}(T) \subset L^{1}(T)$ and $L_{M, \omega}(T)$ is a Banach space with the above norms [1]. This space is called weighted Orlicz space. If we take $M(x, p):=x^{p}, 1<p<\infty$, then this space becomes the weighted Lebesgue space $L^{p}(T, \omega)$.

A weight function $\omega: T \rightarrow[0, \infty]$ belongs to the Muckenhoupt class $A_{p}[14], 1<p<\infty$ if

$\left(\frac{1}{|I|} \int_{I} \omega(x) d x\right)\left(\frac{1}{|I|} \int_{I} \omega^{\frac{1}{1-p}}(x) d x\right)^{p} \leq C$

with a finite constant $C$ independent of the interval $I$ with lenght $\leq 2 \pi$, where $|I|$ denotes the length of $I$.

Without losing generality, suppose that the Fourier series of $f$ is

$$
\sum_{k=1}^{\infty} c_{k}(f) e^{i k x}=: \sum_{k=1}^{\infty} A_{k}(f, x),
$$

where $c_{k}(f), a_{v}(f)$ and $b_{v}(f)$ is the Fourier coefficients of the function $f \in L_{M, \omega}(T)$.

Let $S_{n}(f, x),(n=0,1,2, \ldots)$ be the $n$th partial sum of the series (1.1) at the point $x$, that is,

$S_{n}(f, x):=\sum_{k=1}^{n} A_{k}(f, x)$.

In [1], it was proved that the operator $S_{n}: L_{M, \omega}(T) \rightarrow L_{M, \omega}(T)$ is bounded in $L_{M, \omega}(T)$ if $M \in \Phi_{p}, p>1, \omega \in A_{p}$ and $f \in L_{M, \omega}(T)$. Hence we have [1]

$\left\|S_{n}(f)\right\|_{(M), \omega} \leq C\|f\|_{(M), \omega} \quad n=0,1,2, \ldots$ 
and

$\left\|f-S_{n}(f)\right\|_{(M), \omega} \leq C . E_{n}(f)_{(M), \omega} \quad n=0,1,2, \ldots$

The set of trigonometric polynomials is a dense subset of $L_{\mathrm{M}, \omega}(\mathrm{T})$ since the hypothesis of Lemma 3 of [10] are fulfilled for $M \in \Phi_{p}, p>1, \omega \in A_{p}$.

The well-known Steklov's mean operator is defined as

$\left(\sigma_{t} f\right)(x):=\frac{1}{2 t} \int_{-t}^{t} f(x+u) d u, 0<t<\pi, x \in T$

If $M \in \Phi_{p}, p>1, \omega \in A_{p}$, it was proved that the Hardy Littlewood Maximal function is bounded in $L_{M, \omega}(T)$ [1]. So, the operator $\sigma_{t}$ is bounded in $L_{M, \omega}(T)$ under conditions $M \in \Phi_{p}, p>1, \omega \in A_{p}$. For $x, t \in T, r>0$ and $L_{M, \omega}(T)$ we define

$\sigma_{t}^{r} f(x):=\left(I-\sigma_{t}\right)^{r} f(x)=\sum_{k=0}^{\infty}(-1)^{k}\left[C_{k}^{r}\right] \frac{1}{(2 t)^{k}} \int_{-t}^{t} \ldots \int_{-t}^{t} f\left(x+u_{1}+u_{2+} \ldots+u_{k}\right) d u_{1} \ldots d u_{k}$

where $\left[C_{k}^{r}\right]:=\frac{r \cdot(r-1) \ldots(r-k+1)}{k !}$ for $k>1,\left[C_{1}^{r}\right]:=r$ and $\left[C_{0}^{r}\right]=1$ are binomial coefficients. From the inequality

$\left|\left[C_{k}^{r}\right]\right| \leq \frac{c}{k^{r+1}}, \quad k \in Z^{+}$

we get

$\sum_{k=0}^{\infty}\left|\left[C_{k}^{r}\right]\right|<\infty$

and we have

$\left\|\sigma_{t}^{r} f(x)\right\|_{M, \omega} \leq c\|f\|_{M, \omega}<\infty$

if $M \in \Phi_{p}, p>1$ and , $\omega \in A_{p}$.

The fractional modulus of smoothness of index $r>0$ for $L_{M, \omega}(T)$ is defined as

$\Omega_{M, \omega}^{r}(f, \delta):=\sup _{0<h_{i}, t \leq \delta}\left\|\prod_{i=1}^{[r]}\left(\mathrm{I}-\sigma_{t_{i}}\right)\left(\mathrm{I}-\sigma_{t}\right)^{r-[r]} f\right\|_{M, \omega}$

where $[r]$ denotes the integer part of $r$. Since the operator $\sigma_{t}$ is bounded in $L_{M, \omega}(T)$ we have

$\Omega_{M, \omega}^{r}(f, \delta) \leq c\|f\|_{M, \omega}$

if , $\omega \in A_{p}, 1<p<\infty$. 
The sequence of the best approximation number of $f \in L_{M, \omega}(\boldsymbol{T})$ by trigonometric polynomials is defined by

$E_{n}(f)_{M, \omega}=\inf _{T_{n} \in \tau_{n}}\left\|f-T_{n}\right\|_{M, \omega}$

where $\tau_{n}$ is the set of trigonometric polynomial of degree $\leq n$.

Let $\alpha>0$ be given. We define fractional derivative (in the sense of Weyl) of a function $f \in L^{1}(T)$ as

$f^{(\alpha)}(x):=\frac{a_{0}(f)}{2}+\sum_{v=1}^{\infty} v^{\alpha} A_{v}\left(f, x+\frac{\alpha \pi}{2 v}\right)=: \sum_{v=0}^{\infty} A_{v}\left(f^{(\alpha)}, x\right)$.

Let $W_{M, \omega}^{\alpha}, \alpha>0$, be the class of function $f \in L_{M, \omega}(\boldsymbol{T})$ such that $f^{(\alpha)} \in L_{M, \omega}(\boldsymbol{T})$. If $\alpha>$ $0,1<p<\infty$, then $W_{M, \omega}^{\alpha}$ becomes a Banach space with the norm

$\|f\|_{W_{M, \omega}^{\alpha}}:=\|f\|_{L_{M, \omega}}+\left\|f^{(\alpha)}\right\|_{L_{M, \omega}}$.

Let $\left\{\lambda_{v}^{(n)}\right\}, n=0,1, \ldots, v=0,1, \ldots n$ be an arbitrary infinite triangular number matrix. We associate every function $f \in L^{1}(T)$, on the basis of its Fourier expansion, with a polynomial

$R_{n}(f, \lambda):=\frac{a_{0}}{2} \lambda_{0}^{(n)}+\sum_{v=1}^{n} \lambda_{v}^{(n)} A_{v}(f, x)$

Thus, any triangular matrix $\left\{\lambda_{v}^{(n)}\right\}$ determines a method for constructing the polynomials $R_{n}(f, \lambda)$ or, in other words, a specific sequence of polynomial operators $R_{n}(f, \lambda)$ defined on the space $L_{1}(T)$. In this case, it is also said that the matrix $\left\{\lambda_{v}^{(n)}\right\}$ determines a specific method for summation of Fourier series. It is clear that, for every fixed $n$, the operators $R_{n}(f, \lambda)$ are linear. So, these methods are called linear methods (processes) of summation of Fourier series.

In this work, we investigate some problems about approximation to the derivatives of the functions in $f \in L_{M, \omega}(\boldsymbol{T})$ by these linear methods. We estimate the rate of convergence of $R_{n}\left(f^{(\alpha)}, \lambda\right)-f^{(\alpha)}$ to zero in the $L_{M, \omega}(\boldsymbol{T})$ norm by the fractional modulus of smoothness and the best approximation.

In this paper, we will use the following notation

$A(x) \preccurlyeq B(x) \Leftrightarrow \exists c>0: A(x) \leq c B(x)$. 


\section{Main results and discussion}

Theorem 2.1 Let $M \in \Phi_{p}, p>1, \omega \in A_{p}(T)$, and $f \in L_{M, \omega}(\boldsymbol{T})$. We assume that $\sum_{v=1}^{\infty} v^{\alpha-1} E_{v}(f)_{M, \omega}<\infty$

for some $\alpha \in(0, \infty)$. Then for an arbitrary triangular matrix of the numbers $\left\{\lambda_{v}^{(n)}\right\}$ $\left(\lambda_{0}^{(n)}=1, \lambda_{v}^{(n)}=0, v>n, n=0,1,2, \ldots\right)$, we have

$\left\|R_{n}\left(f^{(\alpha)}, \lambda\right)-f^{(\alpha)}\right\|_{M, \omega} \leq \Omega_{M, \omega}^{r}\left(f^{(\alpha)}, \frac{1}{n}\right)$.

Theorem 2.2 Let $M \in \Phi_{p}, p>1, \omega \in A_{p}(T)$, and $f \in L_{M, \omega}(\boldsymbol{T})$. We assume that $\sum_{v=1}^{\infty} v^{\alpha-1} E_{v}(f)_{M, \omega}<\infty$

for some $\alpha \in(0, \infty)$. Then for an arbitrary triangular matrix of the numbers $\left\{\lambda_{v}^{(n)}\right\}$ $\left(\lambda_{0}^{(n)}=1, \lambda_{v}^{(n)}=0, v>n, n=0,1,2, \ldots\right)$, we have

$\left\|R_{n}\left(f^{(\alpha)}, \lambda\right)-f^{(\alpha)}\right\|_{M, \omega} \leq\left(E_{2^{\mu}-1}^{\gamma}\left(f^{(\alpha)}\right)_{M, \omega} \delta_{2^{\mu}, n}^{\gamma}\right)^{\frac{1}{\gamma}}+E_{n}\left(f^{(\alpha)}\right)_{M, \omega}$

where $\gamma=\min (2, p+\varepsilon)$ with a small positive number $\varepsilon$ and

$\delta_{2^{\mu}, n}^{\gamma}:=\sum_{v=2^{\mu}}^{2^{\mu+1}-1}\left|\lambda_{v+1}^{(n)}-\lambda_{v}^{(n)}\right|+\left|1-\lambda_{2^{\mu+1}}^{(n)}\right|$

$\left(2^{m} \leq n<2^{m+1}\right)$.

Corollary 2.1 Let $\lambda_{v}^{(n)}=1-\frac{v}{n+1}$ for $0 \leq v \leq n$ and $\lambda_{v}^{(n)}=0$ for $v>n, n=0,1,2, \ldots$ Then, for the Fejer means, we have

$\left\|R_{n}\left(f^{(\alpha)}, \lambda\right)-f^{(\alpha)}\right\|_{M, \omega} \preccurlyeq \frac{1}{n+1}\left(\sum_{\mu=0}^{m}(\mu+1)^{\gamma-1} E_{\mu}^{\gamma}\left(f^{(\alpha)}\right)_{M, \omega}\right)^{1 / \gamma}$.

Corollary 2.2 Let $\lambda_{v}^{(n)}=1-\frac{v^{k}}{(n+1)^{k}}$ for $0 \leq v \leq n$ and $\lambda_{v}^{(n)}=0$ for $v>n, n=$ $0,1,2, \ldots$. Then, for the Zygmund means, we have

$\left\|R_{n}\left(f^{(\alpha)}, \lambda\right)-f^{(\alpha)}\right\|_{p q, \omega} \preccurlyeq \frac{1}{(n+1)^{k}}\left(\sum_{\mu=0}^{m}(\mu+1)^{\gamma k-1} E_{\mu}^{\gamma}\left(f^{(\alpha)}\right)_{p q, \omega}\right)^{1 / \gamma}$. 
For $\lambda_{v}(r)=r^{v}(0 \leq r<1, v=0,1,2, \ldots)$ we define

$R_{r}(f, \lambda):=\sum_{v=0}^{\infty} \lambda_{v}(r) A_{v}(f, x)$

Theorem 2.3 Let $M \in \Phi_{p}, p>1, \omega \in A_{p}(T)$ and $f \in L_{M, \omega}(\boldsymbol{T})$. We assume that

$\sum_{v=1}^{\infty} v^{\alpha-1} E_{v}(f)_{M, \omega}<\infty$

for some $\alpha \in(0, \infty)$. Then, for the Abel-Poission means, we have

$\left\|R_{r}\left(f^{(\alpha)}, \lambda\right)-f^{(\alpha)}\right\|_{M, \omega} \preccurlyeq\left((1-r) \sum_{v=0}^{\infty} r^{v}(v+1)^{\gamma-1} E_{2^{v}-1}^{\gamma}\left(f^{(\alpha)}\right)_{M, \omega}\right)^{\frac{1}{\gamma}}$

where $\gamma=\min (2, p+\varepsilon)$ with a small positive number $\varepsilon$.

The similar theorems have been proved in diferent function spaces for $\alpha=0$ and $\gamma=1$ $[5,7,8,9,13,18,19,20,21]$.

For the function $f \in L_{M, \omega}(\boldsymbol{T})$, we defined a mean value operator $B_{h} f$

$\left(B_{h} f\right)(x, u):=\frac{1}{2 h} \int_{-h}^{h} f(x+t u) d t, \quad 0<h<\pi, \quad x \in T, \quad-\infty<u<\infty$.

For $M \in \Phi_{p}, p>1, \omega \in A_{p}(T)$, this operator is a bounded linear operator [1]. By means of the operator $B_{h}$ we define a convolution operator

$\int_{-\infty}^{\infty}\left(B_{h} f\right)(., u) d \mu(u)$

with a bounded variation function $\mu(u)$ on the real line and denote

$D(f, \mu, h, M):=\left\|\int_{-\infty}^{\infty}\left(B_{h} f\right)(., u) d \mu(u)\right\|_{M, \omega}$.

In the approximation theory, the convolution operators are widely used. This type operators have important applications in this theory. Especially, these operators are very useful in constructing approximating polynomials in trigonometric approximation. In different function spaces, evaluating these operators with the best approximation numbers is an important problem in the approximation theory. Now, we investigate the relations between these transforms and the best approximations in the weighted Orlicz spaces.

Theorem 2.4 Let $M \in \Phi_{p}, p>1, \omega \in A_{p}(T)$, and $f \in L_{M, \omega}(\boldsymbol{T})$. We assume that 
$\sum_{v=1}^{\infty} v^{\alpha-1} E_{v}(f)_{M, \omega}<\infty$

for some $\alpha \in(0, \infty)$. Then, for every natural number $m$

$D\left(f^{(\alpha)}, \mu, h, M\right) \preccurlyeq \sum_{r=0}^{m}\left(E_{2^{r}}^{\gamma}\left(f^{(\alpha)}\right)_{M, \omega} \delta_{2^{r}, h}^{\gamma}\right)^{\frac{1}{\gamma}}+E_{2^{m+1}}\left(f^{(\alpha)}\right)_{M, \omega}$

where $\gamma=(2, p+\varepsilon)$ with a small positive number $\varepsilon$ and

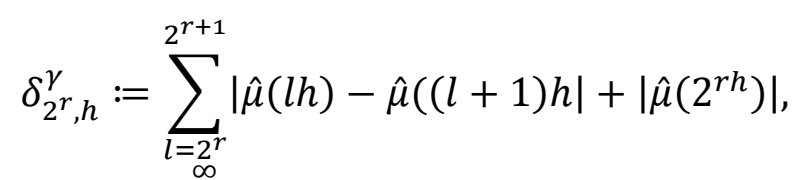

$\hat{\mu}(x):=\int_{-\infty}^{\infty} \frac{\operatorname{sinux}}{u x} d \mu(u), \quad 0<h \leq \pi$

Theorem 2.5 Let $M \in \Phi_{p}, p>1, \omega \in A_{p}(T)$, and $f \in L_{M, \omega}(\boldsymbol{T})$. We assume that

$\sum_{v=1}^{\infty} v^{\alpha-1} E_{v}(f)_{M, \omega}<\infty$

for some $\alpha \in(0, \infty)$. Suppose that the function $F(x)$ satifies the conditions

$\|F(x)\| \leq c_{1}, \sum_{k=2^{\mu}}^{2^{\mu+1}-1} \mid F(k h)-F\left((k+1) h \mid \leq c_{2}, \quad h \leq 2^{-m-1}\right.$

with some constants $c_{1}, c_{2}$. If $\hat{\mu}_{1}, \hat{\mu}_{2}$ are the functions satisfying the condition

$\hat{\mu}_{1}(x)=\hat{\mu}_{2}(x) F(x), \quad|x|<1$

then

$D\left(f^{(\alpha)}, \mu_{1}, h, M\right) \preccurlyeq D\left(f^{(\alpha)}, \mu_{2}, h, M\right)+E_{2^{m+1}}\left(f^{(\alpha)}\right)_{M, \omega}$.

In [15], the convolution operator was defined by means of the usual shift $f(x+t)$ and these theorems were proved in Orlicz spaces with $\alpha=0, \gamma=1$. Using the operator $B_{h}$ these theorems were proved in weighted Orlicz spaces, in which the Young function is convex, in [24] with $\alpha=0, \gamma=1$. This problem was also investigated in weighted Lorentz spaces [23] and variable exponent Lebesgue spaces [6] with $\alpha=0, \gamma=1$. The similar problem was investigated in [4] with $\alpha=0$. 


\section{Auxiliary results}

We give multiplier theorem and Littlewood-Paley theorem in the space $L_{M, \omega}(\boldsymbol{T})$.

Theorem 3.1 [1] Let a sequence $\xi_{k}$ satisfy the conditions

$$
\left|\xi_{k}\right| \leq A, \sum_{k=2^{j-1}}^{2^{j}-1}\left|\xi_{k}-\xi_{k+1}\right| \leq A
$$

where $A>0$ is independent of $k$ and $j$. If $M \in \Phi_{p}, p>1, \omega \in A_{p}(T)$, and $f \in$ $L_{M, \omega}(\boldsymbol{T})$ then there exists a function $F \in L_{M, \omega}(\boldsymbol{T})$ such that the series

$\frac{\lambda_{0} a_{0}}{2}+\sum_{k=0}^{\infty} \lambda_{k}\left(a_{k}(f) \cos k x+b_{k}(f) \sin k x\right)$

is Fourier series for $F$ and the inequality

$\|F\|_{M, \omega} \leq C A\|f\|_{M, \omega}$

holds with some constant $C$ independent of $f$.

Theorem $3.2[1] M \in \Phi_{p}, p>1, \omega \in A_{p}(T)$, and $f \in L_{M, \omega}(\boldsymbol{T})$ there exist constants $C>0$ and $c>0$ depending only on $M$ and $\omega$ such that

$c\|f\|_{M, \omega} \leq\left\|\left(\sum_{j=0}^{\infty}\left|\sum_{k=2^{j-1}}^{2^{j}-1} A_{k}(x, f)\right|^{2}\right)\right\|_{M, \omega}^{\frac{1}{2}} \leq C\|f\|_{M, \omega}$.

\section{Proofs of main results}

Proof of Theorem 2.1 Let $2^{m} \leq h<2^{m+1}$. Under the conditions of the theorem, we have $f \in \mathcal{W}_{M, \omega}^{(\alpha)}(\boldsymbol{T})[1]$. Let

$$
S_{n}\left(f^{(\alpha)}, x\right):=\sum_{v=1}^{n} A_{v}\left(f^{(\alpha)}, x\right)
$$

be the partial some of Fourier series of the function $f^{(\alpha)}$. By the properties of the norm, we have

$$
\left\|R_{n}\left(f^{(\alpha)}, \lambda\right)-f^{(\alpha)}\right\|_{M, \omega}=\left\|\sum_{v=0}^{n} \lambda_{v}^{(n)} A_{v}\left(f^{(\alpha)}, x\right)-f^{(\alpha)}(x)\right\|_{M, \omega}
$$


$\leq\left\|\sum_{v=0}^{n}\left(1-\lambda_{v}^{(n)}\right) A_{v}\left(f^{(\alpha)}, x\right)\right\|_{M, \omega}+\left\|\sum_{v=n+1}^{\infty} A_{v} f^{(\alpha)}(x)\right\|_{M, \omega}=: I_{1}+I_{2}$.

From [4, Lemma 2.1] and [1, Th 1.5 and Th.1.1] we get

$I_{2}=\left\|\sum_{v=n+1}^{\infty} A_{v}\left(f^{(\alpha)}, x\right)\right\|_{M, \omega} \preccurlyeq E_{n}\left(f^{(\alpha)}\right)_{M, \omega} \preccurlyeq \Omega_{M, \omega}^{r}\left(f^{(\alpha)}, \frac{1}{n}\right)$.

Now we estimate the norm

$I_{1}=\left\|\sum_{v=0}^{n} \frac{1-\lambda_{v}^{(n)}}{\left(1-\frac{\sin \frac{v}{n}}{\frac{v}{n}}\right)^{r}} A_{v}\left(f^{(\alpha)}, x\right)\left(1-\frac{\sin \frac{v}{n}}{\frac{v}{n}}\right)^{r}\right\|_{M, \omega}$.

Let

$\mu_{v, r}^{(n)}:=\left\{\begin{array}{cc}\frac{\left(1-\lambda_{v}^{(n)}\right)}{\left(1-\frac{\sin \frac{v}{n}}{\frac{v}{n}}\right)^{r}}, & v \leq n, \\ 0 & v>n .\end{array}\right\}$

For the sequence $\left\{\mu_{v, r}^{(n)}\right\}$ the conditions of Theorem 3.1 are satisfied. Applying Theorem 3.1 and the proof method in [1, Th. 1.9] we get

$$
\begin{aligned}
& I_{1}=\left\|\sum_{v=0}^{n} \mu_{v, r}^{(n)} A_{v}\left(f^{(\alpha)}, x\right)\left(1-\frac{\sin \frac{v}{n}}{\frac{v}{n}}\right)^{r}\right\|_{M, \omega} \preccurlyeq\left\|\sum_{v=0}^{n} A_{v}\left(f^{(\alpha)}, x\right)\left(1-\frac{\sin \frac{v}{n}}{\frac{v}{n}}\right)^{r}\right\|_{M, \omega} \\
& \leq\left\|\left(I-\sigma_{1 / n}\right)^{r} f^{(\alpha)}\right\|_{M, \omega}=\left\|\sum_{v=0}^{n}\left(I-\sigma_{1 / n}\right)^{[r]}\left(I-\sigma_{1 / n}\right)^{r-[r]} f^{(\alpha)}\right\|_{M, \omega} \\
& \leqslant \sup _{0<h_{i}, t \leq \frac{1}{n}}\left\|\prod_{i=1}^{[r]}\left(I-\sigma_{h_{i}}\right)\left(I-\sigma_{t}\right)^{r-[r]} f^{(\alpha)}\right\|_{M, \omega} \leqslant \Omega_{M, \omega}^{r}\left(f^{(\alpha)}, \frac{1}{n}\right)
\end{aligned}
$$

This proves Theorem 2.1.

Proof of Theorem 2.2 Let $2^{m} \leq h<2^{m+1}$. We have

$$
\left\|R_{n}\left(f^{(\alpha)}, \lambda\right)-f^{(\alpha)}\right\|_{M, \omega}=\left\|\sum_{v=0}^{n} \lambda_{v}^{(n)} A_{v}\left(f^{(\alpha)}, x\right)-\left(f^{(\alpha)}, x\right)\right\|_{M, \omega}
$$




$$
\begin{aligned}
\leq\left\|\sum_{v=0}^{n}\left(1-\lambda_{v}^{(n)}\right) A_{v}\left(f^{(\alpha)}, x\right)\right\|_{M, \omega}+\left\|\sum_{v=n+1}^{\infty} A_{v}\left(f^{(\alpha)}, x\right)\right\|_{M, \omega} \\
\leqslant\left\|\sum_{v=1}^{n}\left(1-\lambda_{v}^{(n)}\right) A_{v}\left(f^{(\alpha)}, x\right)\right\|_{M, \omega}+E_{n}\left(f^{(\alpha)}\right)_{M, \omega} .
\end{aligned}
$$

From Theorem 3.2, we get

$$
\left\|\sum_{v=1}^{n}\left(1-\lambda_{v}^{(n)}\right) A_{v}\left(f^{(\alpha)}, x\right)\right\|_{M, \omega} \leq c\left\|\left(\sum_{\mu=0}^{m}\left|\sum_{v=2^{\mu}}^{2^{\mu+1}-1}\left(1-\lambda_{v}^{(n)}\right) A_{v}\left(f^{(\alpha)}, x\right)\right|^{2}\right)^{\frac{1}{2}}\right\|_{M, \omega}
$$

Using the Abel transformation, we obtain

$$
\begin{gathered}
\sigma_{n, \mu}(x):=\sum_{v=2^{\mu}}^{2^{\mu+1}-1}\left(1-\lambda_{v}^{(n)}\right) A_{v}\left(f^{(\alpha)}, x\right) \\
=\sum_{v=2^{\mu}}^{2^{\mu+1}-1}\left(S_{v}(f, x)-S_{2^{\mu}-1}(f, x)\right)\left(\lambda_{v+1}^{(n)}-\lambda_{v}^{(n)}\right) \\
+\left(S_{2^{\mu+1}-1}(f, x)-S_{2^{\mu}-1}(f, x)\right)\left(1-\lambda_{2^{\mu+1}}^{(n)}\right) .
\end{gathered}
$$

From Minkowski's inequality and the monotonicity of the sequence of the best approximation numbers, we get

$$
\begin{aligned}
& \left\|\sigma_{n, \mu}(x)\right\|_{M, \omega} \leq \sum_{v=2^{\mu}}^{2^{\mu+1}-1}\left\|S_{v}(f, x)-S_{2^{\mu}-1}(f, x)\right\|_{M, \omega}\left|\lambda_{v+1}^{(n)}-\lambda_{v}^{(n)}\right| \\
& +\left\|S_{2^{\mu+1}-1}(f, x)-S_{2^{\mu}-1}(f, x)\right\|_{M, \omega}\left|1-\lambda_{2^{\mu+1}}^{(n)}\right| \\
& \leq c E_{2^{\mu}-1}(f)_{M, \omega}\left(\sum_{v=2^{\mu}}^{2^{\mu+1}-1}\left|\lambda_{v+1}^{(n)}-\lambda_{v}^{(n)}\right|+\left|1-\lambda_{2^{\mu+1}}^{(n)}\right|\right) \\
& \leq c E_{2^{\mu}-1}(f)_{M, \omega} \delta_{2^{\mu}, n} .
\end{aligned}
$$

On the other hand, we have [1]

$$
\left\|\left(\sum_{\mu=0}^{m}\left|\sigma_{n, \mu}(x)\right|^{2}\right)^{1 / 2}\right\|_{M, \omega} \leq c\left(\sum_{\mu=0}^{m}\left\|\sigma_{n, \mu}(x)\right\|^{\gamma}\right)^{1 / \gamma},
$$

where $\gamma=\min (2, p+\varepsilon)$ with a small positive number $\varepsilon$. Therefore, we obtain the required inequality 


$$
\left\|\sum_{v=0}^{n}\left(1-\lambda_{v}^{(n)}\right) A_{v}\left(f^{(\alpha)}, x\right)\right\|_{M, \omega} \leq c\left(\sum_{\mu=0}^{m} E_{2^{\mu-1}}^{\gamma}\left(f^{(\alpha)}\right)_{M, \omega} \cdot \delta_{2^{\mu}, n}^{\gamma}\right)^{1 / \gamma}
$$

Proof of Theorem 2.3 Let $2^{m} \leq n=\left[\frac{1}{1-r}\right]<2^{m+1}$ and $\lambda_{v}(r)=r^{v}, 0 \leq r<1, v=$ $0,1,2, \ldots$ We have

$$
\begin{aligned}
& \left\|R_{r}\left(f^{(\alpha)}, \lambda\right)-f^{(\alpha)}\right\|_{M, \omega}=\left\|\sum_{v=0}^{\infty}\left(1-r^{v}\right) A_{v}\left(f^{(\alpha)}, x\right)\right\|_{M, \omega} \\
& \left\|\sum_{v=0}^{2^{m+1}-1}\left(1-r^{v}\right) A_{v}\left(f^{(\alpha)}, x\right)\right\|_{M, \omega}+\left\|\sum_{v=2^{m+1}-1}^{\infty}\left(1-r^{v}\right) A_{v}\left(f^{(\alpha)}, x\right)\right\|_{M, \omega}=: I_{1}+I_{2} .
\end{aligned}
$$

It is easily seen that the conditions of Theorem 3.1 are satisfied for the sequence $\left\{1-r^{\nu}\right\}$. Then, by Theorem 3.1, [4, Lemma 2.1] and [1, Th.1.5] we have

$I_{2} \leq c\left\|\sum_{v=2^{m+1}}^{\infty} A_{v}\left(f^{(\alpha)}, x\right)\right\|_{M, \omega} \leq c E_{n}\left(f^{(\alpha)}\right)_{M, \omega}$.

From Theorem 3.2 and (4.1)

$$
\begin{aligned}
I_{1} & \leq\left\|\left(\sum_{v=0}^{m}\left|\sum_{\mu=2^{v}}^{2^{v+1}-1}\left(1-r^{\mu}\right) A_{\mu}\left(f^{(\alpha)}, x\right)\right|^{2}\right)^{\frac{1}{2}}\right\|_{M, \omega} \\
& \leq\left(\sum_{v=0}^{m}\left\|\sum_{\mu=2^{v}}^{2^{v+1}-1}\left(1-r^{\mu}\right) A_{\mu}\left(f^{(\alpha)}, x\right)\right\|_{M, \omega}^{\gamma}\right)^{\frac{1}{\gamma}} .
\end{aligned}
$$

Applying Abel's transform and Theorem 3.1, we get

$$
\begin{aligned}
& \left\|\sum_{\mu=2^{v}}^{2^{v+1}-1}\left(1-r^{\mu}\right) A_{\mu}\left(f^{(\alpha)}, x\right)\right\|_{M, \omega} \\
& \leq \sum_{\mu=2^{v}}^{2^{v+1}-1}\left\|S_{\mu}\left(f^{(\alpha)}, x\right)-S_{2^{v}-1}\left(f^{(\alpha)}, x\right)\right\|_{M, \omega}\left|r^{\mu+1}-r^{\mu}\right| \\
& \quad+\left\|S_{2^{v+1}-1}\left(f^{(\alpha)}, x\right)-S_{2^{v}-1}\left(f^{(\alpha)}, x\right)\right\|_{M, \omega}\left|1-r^{2^{v+1}}\right| \\
& \leq c E_{2^{v}-1}\left(f^{(\alpha)}\right)_{M, \omega} 2^{v+1}(1-r) .
\end{aligned}
$$

From the monotonicity of the best approximation, we have 


$$
\begin{aligned}
& \left\|\sum_{\mu=2^{v}}^{2^{v+1}-1}\left(1-r^{\mu}\right) A_{\mu}\left(f^{(\alpha)}, x\right)\right\|_{M, \omega}^{\gamma} \leq c E_{2^{v}-1}^{\gamma}\left(f^{(\alpha)}\right)_{M, \omega} 2^{\gamma(v+1)}(1-r)^{\gamma} \\
& \leq c(1-r)^{\gamma} \sum_{\mu=2^{v-1}}^{2^{v}-1} E_{2^{v}-1}^{\gamma}\left(f^{(\alpha)}\right)_{M, \omega} \cdot
\end{aligned}
$$

Then we get

$$
\begin{aligned}
& I_{1} \leq c\left((1-r)^{\gamma}\left\|A_{1}\left(f^{(\alpha)}, x\right)\right\|_{M, \omega}^{\gamma}(1-r)^{\gamma} \sum_{\mu=2^{v-1}}^{2^{v-1}} \mu^{\gamma-1} E_{\mu}^{\gamma}\left(f^{(\alpha)}\right)_{M, \omega}\right)^{\frac{1}{\gamma}} \\
& \leq c\left((1-r) \sum_{\mu=0}^{n}(\mu+1)^{\gamma-1} E_{\mu}^{\gamma}\left(f^{(\alpha)}\right)_{M, \omega}\right)^{1 / \gamma} .
\end{aligned}
$$

Therefore, we have

$$
\begin{aligned}
& \left\|R_{r}\left(f^{(\alpha)}, \lambda\right)-f^{(\alpha)}\right\|_{M, \omega} \leq c(1-r)\left(\sum_{\mu=0}^{n}(\mu+1)^{\gamma-1} E_{\mu}^{\gamma}\left(f^{(\alpha)}\right)_{M, \omega}\right)^{1 / \gamma}+E_{n}\left(f^{(\alpha)}\right)_{M, \omega} \\
& \leq c(1-r)\left(\sum_{\mu=0}^{n} r^{\mu}(\mu+1)^{\gamma-1} E_{\mu}^{\gamma}\left(f^{(\alpha)}\right)_{M, \omega}\right)^{1 / \gamma} .
\end{aligned}
$$

Proof of Theorem 2.4 Let $m \in \mathbb{N}, 2^{m} \leq h<2^{m+1}$. Under the conditions of the theorem, we have $f \in \mathcal{W}_{M, \omega}^{(\alpha)}(\boldsymbol{T})[1]$. We suppose that

$S_{2^{m+1}} f^{(\alpha)}:=\sum_{k=1}^{2^{m+1}} c_{k}\left(f^{(\alpha)}\right) e^{i k x}$

is the partial sum of Fourier series of the function $f^{(\alpha)}$. From the definition of the quantity $D\left(f^{(\alpha)}, \mu, h, M\right)$ and the properties of the norm we have

$$
\begin{aligned}
& D\left(f^{(\alpha)}, \mu, h, M\right)=\left\|\int_{-\infty}^{\infty}\left(B_{h} f^{(\alpha)}\right)(x, u) d \mu(u)\right\|_{M, \omega} \\
& \leq\left\|\int_{-\infty}^{\infty}\left[\left(B_{h} f^{(\alpha)}\right)(x, u)-\left(B_{h} S_{2^{m+1}} f^{(\alpha)}\right)(x, u)\right] d \mu(u)\right\|_{M, \omega} \\
& +\left\|\int_{-\infty}^{\infty}\left(B_{h} S_{2^{m+1}} f^{(\alpha)}\right)(x, u) d \mu(u)\right\|_{M, \omega} .
\end{aligned}
$$

By the boundedness of the operator $B_{h}$ and [1,Th.1.5], we obtain 


$$
\begin{aligned}
\| & \int_{-\infty}^{\infty}\left[\left(B_{h} f^{(\alpha)}\right)(x, u)-\left(B_{h} S_{2^{m+1}} f^{(\alpha)}\right)(x, u)\right] d \mu(u) \|_{M, \omega} \\
\leq c & \int_{-\infty}^{\infty}\left\|\left(B_{h} f^{(\alpha)}\right)(x, u)-\left(B_{h} S_{2^{m+1}} f^{(\alpha)}\right)(x, u)\right\|_{M, \omega} d \mu(u) \\
= & \int_{-\infty}^{\infty}\left\|\left(B_{h}\left(f^{(\alpha)}-\sigma_{h} S_{2^{m+1}} f^{(\alpha)}\right)(x, u)\right)\right\|_{M, \omega} d \mu(u) \\
\leq c & \int_{-\infty}^{\infty}\left\|f^{(\alpha)}-S_{2^{m+1}} f^{(\alpha)}\right\|_{M, \omega} d \mu(u) \\
= & \int_{-\infty}^{\infty}\left\|f^{(\alpha)}-S_{2^{m+1}} f^{(\alpha)}\right\|_{M, \omega} d \mu(u) \\
= & \int_{-\infty}^{\infty}\left\|f^{(\alpha)}-S_{2^{m+1}}^{(\alpha)}\right\|_{M, \omega} d \mu(u) \\
\leq & c E_{2^{m+1}}\left(f^{(\alpha)}\right)_{M, \omega}
\end{aligned}
$$

So, we have

$D\left(f^{(\alpha)}, \mu, h, M\right) \leq\left\|\int_{-\infty}^{\infty}\left(\sigma_{h} S_{2^{m+1}} f^{(\alpha)}\right)(x, u) d \mu(u)\right\|_{M, \omega}+c E_{2^{m+1}}\left(f^{(\alpha)}\right)_{M, \omega}$.

Then we get

$$
\begin{aligned}
& \int_{-\infty}^{\infty}\left(B_{h} S_{2^{m+1}} f^{(\alpha)}\right)(x, u) d \mu(u)=\int_{-\infty}^{\infty}\left(\frac{1}{2 h} \int_{-h}^{h} S_{2^{m+1}} f^{(\alpha)}(x+\mathrm{t} u) d t\right) d \mu(u) \\
= & \int_{-\infty}^{\infty}\left(\frac{1}{2 h} \int_{-h}^{h} \sum_{r=1}^{2^{m+1}} c_{r}\left(f^{(\alpha)}\right) e^{i r(x+\mathrm{t} u)} d t\right) d \mu(u) \\
= & \int_{-\infty}^{\infty}\left(\frac{1}{2 h} \sum_{r=1}^{2^{m+1}} c_{r}\left(f^{(\alpha)}\right) e^{i r \mathrm{x}} \int_{-h}^{h} e^{i r \mathrm{tu}} d t\right) d \mu(u) \\
= & \sum_{r=1}^{2^{m+1}} c_{r}\left(f^{(\alpha)}\right) e^{i r \mathrm{x}} \int_{-\infty}^{\infty} \frac{e^{i r \mathrm{hu}}-e^{-i r h u}}{2 i r h u} d \mu(u) \\
= & \sum_{r=1}^{2^{m+1}} A_{r}\left(f^{(\alpha)}, x\right) \hat{\mu}(r h) .
\end{aligned}
$$

Therefore, we have 


$$
D\left(f^{(\alpha)}, \mu, h, M\right) \preccurlyeq\left\|\sum_{r=1}^{2^{m+1}} A_{r}\left(f^{(\alpha)}, x\right) \hat{\mu}(r h)\right\|_{M, \omega}+E_{2^{m+1}}\left(f^{(\alpha)}\right)_{M, \omega} .
$$

From Theorem 3.2 and [1], we obtain

$$
\begin{aligned}
& \left\|\sum_{r=1}^{2^{m+1}} A_{r}\left(f^{(\alpha)}, x\right) \hat{\mu}(r h)\right\|_{M, \omega} \leqslant\left\|\left(\sum_{r=0}^{m}\left|\sum_{l=2^{r}}^{2^{r+1}} A_{l}(x) \hat{\mu}(l h)\right|^{2}\right)^{\frac{1}{2}}\right\|_{M, \omega} \\
& :=c\left\|\left(\sum_{r=0}^{m} \Delta_{r, \mu}^{2}\right)^{\frac{1}{2}}\right\|_{M, \omega} \leqslant c\left\|\sum_{r=0}^{m}\left(\Delta_{r, \mu}^{2}\right)^{\frac{1}{2}}\right\|_{M, \omega} \\
& \left\|\left(\sum_{r=0}^{m} \Delta_{r, \mu}^{2}\right)^{\frac{1}{2}}\right\|_{M, \omega}^{m} \leq c \sum_{r=0}^{m}\left(\left\|\Delta_{r, \mu}^{\gamma}\right\|\right)_{M, \omega}^{1 / \gamma} .
\end{aligned}
$$

Applying the Abel transform to $\Delta_{r, \boldsymbol{\mu}}$, we get

$$
\begin{gathered}
\Delta_{r, \mu}=\sum_{l=2^{r}}^{2^{r+1}}\left[S_{l} f^{(\alpha)}(x)-S_{2^{r+1}} f^{(\alpha)}(x)\right](\hat{\mu}(l h)-\hat{\mu}((l+1) h) \\
+\left[S_{2^{r+1}} f^{(\alpha)}(x)-S_{2^{r}} f^{(\alpha)}(x)\right] \hat{\mu}\left(2^{r} h\right) .
\end{gathered}
$$

From [1, Th. 1.5],

$$
\begin{aligned}
& \left\|\Delta_{r, \mu}\right\|_{M, \omega}=\sum_{l=2^{r}}^{2^{r+1}}\left\|S_{l} f^{(\alpha)}(x)-S_{2^{r+1}} f^{(\alpha)}(x)\right\|_{M, \omega}(\hat{\mu}(l h)-\hat{\mu}((l+1) h) \\
& +\| S_{2^{r+1} f^{(\alpha)}(x)-S_{2^{r}} f^{(\alpha)}(x) \|_{M, \omega} \hat{\mu}\left(2^{r} h\right)}^{\leq E^{r}{ }_{2^{r}}\left(f^{(\alpha)}\right)_{M, \omega} \cdot \delta^{r}{ }_{2^{r}, h} .}
\end{aligned}
$$

Then

$$
\left\|\sum_{r=1}^{2^{m+1}-1} A_{r}\left(f^{(\alpha)}\right) e^{i r \mathrm{x}} \hat{\mu}(r h)\right\|_{M, \omega} \leq c\left(\sum_{r=0}^{m} E^{\gamma}{ }^{r-1}\left(f^{(\alpha)}\right)_{M, \omega} \cdot \delta^{\gamma}{ }^{r}, h\right)^{\frac{1}{\gamma}} .
$$

This completes the proof.

Proof of Theorem 2.5 Under the conditions of the theorem, we have $f \in \mathcal{W}_{M, \omega}^{(\alpha)}(\boldsymbol{T})$ [1].We have 


$$
D\left(f^{(\alpha)}, \mu_{1}, h, M\right) \leq\left\|\int_{-\infty}^{\infty}\left(B_{h} S_{2^{m+1}} f^{(\alpha)}\right)(x, u) d \mu(u)\right\|_{M, \omega}+E_{2^{m+1}}\left(f^{(\alpha)}\right)_{M, \omega}
$$

From the the properties of the function and Theorem 3.1, we obtain

$$
\begin{aligned}
& \left\|\int_{-\infty}^{\infty}\left(B_{h} S_{2^{m+1}} f^{(\alpha)}\right)(x, u) \mathrm{d} \mu_{1}(u)\right\|_{M, \omega}=\left\|\sum_{r=1}^{2^{m+1}} c_{r}\left(f^{(\alpha)}\right) e^{i r x} \mu_{1}(r h)\right\|_{M, \omega} \\
& =\left\|\sum_{r=1}^{2 m+1} c_{r}\left(f^{(\alpha)}\right) e^{i r x} \mu_{2}(r h) F(r h)\right\|_{M, \omega} \\
& \leq c\left\|\sum_{r=1}^{2^{m+1}} c_{r}\left(f^{(\alpha)}\right) e^{i r x} \mu_{2}(r h)\right\|_{M, \omega}=\left\|\int_{-\infty}^{\infty}\left(B_{h} S_{2^{m+1}} f^{(\alpha)}\right)(x, u) d \mu_{2}(u)\right\|_{M, \omega} \\
& =\left\|S_{2^{m+1}} \int_{-\infty}^{\infty}\left(B_{h} f^{(\alpha)}\right)(x, u) d \mu_{2}(u)\right\|_{M, \omega} \leq \boldsymbol{c}\left\|\int_{-\infty}^{\infty}\left(B_{h} f^{(\alpha)}\right)(x, u) d \mu_{2}(u)\right\|_{M, \omega} .
\end{aligned}
$$

This completes the proof.

\section{References}

[1] Akgün, R., Some inequalities of trigonometric approximation in weighted Orlicz spaces, Mathematica Slovaca, 66, 1, 217-234, (2016).

[2] Akgün, R. and Koç, H., Simultaneous approximation of functions in Orlicz spaces with Muckenhoupt weights, Complex Variables and Elliptic Equations, 61, 8, 1107-1115, (2016).

[3] Chen, Y.M., On two-functional spaces, Studia Mathematica, 24, 61-88, (1964).

[4] Dogu, A., Avsar, A.H. and Yildirir, Y.E., Some inequalities about convolution and trigonometric approximation in weighted Orlicz spaces. Proceeding of the Institute of Mathematics and Mechanics National Academy of Sciences of Azerbaijan, 44, 1, 107-115, (2018).

[5] Gavriljuk, V.G., Linear summation methods for the Fourier series and best approximation, Ukrainian Mathematical Journal, 15, 4, 412-418, (1963).

[6] Israfilov, D.M. and Yirtici, E., Convolutions and best approximations in variable exponent Lebesgue spaces, Mathematical Reports (Bucuresti), 18(68), 4, 497508, (2016).

[7] Jafarov, S.Z., Linear methotds of summing Fourier series and approximation in weight Orlicz spaces, Turkish Journal of Mathematics, 42, 6, 2916-2925, (2018).

[8] Jafarov, S.Z., Approximation by linear means of Fourier series in weighted Orlicz spaces Proceeding of the Institute of Mathematics and Mechanics National Academy of Sciences of Azerbaijan, 43, 2, 175-187, (2017).

[9] Jafarov, S.Z., Approximation by linear means of Fourier series in weighted Lebesque spaces with variable exponents, Ukrainian Mathematical Journal, 66, 10, 1509-1518, (2015). 
[10] Khabazi, M., The mean convergence of trigonometric Fourier series in weighted Orlicz classes, Proceedings of A. Razmadze Mathematical Institute, 129, 6575, (2002).

[11] Krasnoselíski, M.A. and Ruticki, Y.B., Convex functions and Orlicz spaces, Translated from the first Russian edition by Leo F. Boron, P. Noordho Ltd., Groningen, (1961).

[12] Koç, H., Simultaneous approximation by polynomials in Orlicz spaces generated by quasiconvex Young functions. Kuwait J. Science, 43, 4, 18-31, (2016).

[13] Kokilashvili, V. and Tsanava, T., "On the norm estimate of deviation by linear summability means and an extension of the Bernstein inequality, Proceedings of A. Razmadze Mathematical Institute, 154, 144-146 (2010).

[14] Muckenhoupt, B., Weighted norm inequalities for the Hardy maximal function, Transactions of the American Mathematical Society, 165, 207-226, (1972).

[15] Ponomarenko, V.G. and Timan, M.F., The properties of convolution type transforms in the Orlicz spaces, theory of approximation of functions, Proceedings of the Institute of Mathematics and Mechanics, 3, Donetsk, (1998).

[16] Rao, M.M. and Ren, Z.D., Applications of Orlicz spaces, Marcel Dekker Inc., New York, (2002).

[17] Rao, M.M. and Ren, Z.D., Theory of Orlicz spaces, Marcel Dekker Inc., New York, (1991).

[18] Stechkin, S.B., Approximation of periodic functions by Fejíer sums, Trudy Matematicheskogo Instituta imeni V. A. Steklova, 2, 48-60, (1961).

[19] Timan, M.F., Best approximation of a function and linear methods for the summation of Fourier series, Izvestiya Rossiiskoi Akademii Nauk Seriya Matematicheskaya, 29, 587-604, (1965).

[20] Timan, M.F., Approximation of continuous periodic functions by linear operators constructed on the basis of their Fourier series, Doklayd Akademii Nauk. SSSR, 181, 1339-1342, (1968).

[21] Timan, M.F., Some linear summation processes for the Fourier series and the best approximation, Doklayd Akademii Nauk. SSSR, 145, 741-743, (1962).

[22] Yildirir, Y.E. and Cetintas, R., Trigonometric approximation in weighted Orlicz spaces. Proceeding of the Institute of Mathematics and Mechanics National Academy of Sciences of Azerbaijan, 42, 1, 25-37, (2016).

[23] Yildirir, Y.E. and Dogu, A., Approximation in weighted Lorentz spaces, Journal Mathematical Science: Advences and Applications, 54, 1-9, (2018).

[24] Yildirir, Y.E. and Israfilov, D.M., The properties of convolution type transforms in weighted Orlicz spaces, Glasnik Matematicki, 65, 461-474, (2010). 